\title{
Relationship between Respiratory Movements and Energy Efficiency in the Post-Exercise Recovery Phase
}

\author{
Yukari OHASHI $^{1}$, Masashi KAMIOKA ${ }^{1}$ and Ken MATSUOKA ${ }^{2}$ \\ ${ }^{1}$ Ibaraki Prefectural University of Health Sciences, Ibaraki 300-0394, Japan \\ ${ }^{2}$ Tokyo Medical University, Tokyo 160-8402, Japan
}

\begin{abstract}
The breathing movement is highly automated, but it can be voluntarily controlled in some situations. Compared with period of exercise and post-exercise, subjects could control breathing more voluntarily in the post-exercise recovery phase. So, we analyzed relationship between the strategy for controlling the breathing movement pattern and energy efficiency in this phase. Fifteen healthy men (mean age, 22.1 years) were subjected to exercise with a bicycle ergometer at $100 \mathrm{~W}$ for 5 minutes, then respiratory pattern was measured four times for 5 minutes after exercise. The measurement items were 1) chest expansion, 2) abdomen expansion, 3) tidal volume, 4) respiration rate, 5) minute ventilation, 6) oxygen intake, and 7) heart rate. The items 1) and 2) were measured by a three-dimensional motion analysis system, and the items 3) through 7) were measured by a respiratory gas analyzer. As for the breathing movement pattern, there were differences among the subjects in terms of the rates of increase in chest expansion and in respiration rate after exercise. The subjects were classified into 4 groups according to these differences. The group, in which respiration rate was increased, showed the marked increase in minute ventilation and oxygen intake, and the rapid recovery of heart rate. The group, in which chest expansion rate was increased, on the other hand, showed small increase in minute ventilation and oxygen intake, and the slow recovery of heart rate. The breathing strategies which are beneficial to energy efficiency exist, however, healthy men do not always select the beneficial strategies.
\end{abstract}

Key words: respiratory movements, respiratory gas analysis, energy efficiency

(J Jpn Phys Ther Assoc 4: 7-11, 2001)

$\mathbf{R}$ espiratory movements of healthy person can be voluntarily controlled in some situations, though they are highly automated. In this regard, breathing has the characteristics similar to those of walking. According to a study of walking, the best energy efficiency is induced by free walking at the cadence that is unconsciously selected ${ }^{1}$. The stride and cadence, which are designed for walking at the constant speed, can be traded off with each other, but the strategy for walking, i.e., optimal cadence, is to exist when a viewpoint of energy efficiency is also taken into consideration. In respiratory movements, a trade-off of tidal volume with respiration rate may be allowed to obtain the constant minute ventilation. A trade-off of the chest

Received: July 18, 2000

Accepted: February 4, 2001

Correspondence to: Yukari Ohashi, Department of Physical Therapy, School of Medical Health, Ibaraki Prefectural University of Health Sciences, 4669-2 Ami, Ami-Machi, Inashiki-Gun, Ibaraki 300-0394, Japan breathing with the abdominal breathing is also allowed to obtain the constant tidal volume. Physiologically, there are regional differences in ventilation and pulmonary blood flow during respiration in healthy persons at rest in a standing position ${ }^{2}$. The strategy for controlling respiratory movements is restricted to a certain degree. Nevertheless, many points remain unsolved regarding details of respiratory movement patterns. From a kinesiological point of view as well, detailed classification of respiratory movement patterns, individual differences in the patterns, and relationships between the patterns and oxygen intake remain unsolved.

Conventional studies have shown the respiratory movement patterns during exercise changing along with the increase in intensity of exercise as follows: mainly tidal volume is increased by slight exercise, both tidal volume and respiration rate are increased by moderate exercise, and mainly respiration rate is increased by hard exercise ${ }^{3)}$. 
However, details of the trade-off of tidal volume with respiration rate during moderate exercise have not been clarified. In recent years, some reports have shown studies on changes in difference of expansion between the chest and abdomen during exercise and a relationship between tidal volume and respiration rate by means of a mercury strain gauge and plethysmograph ${ }^{4-6)}$. In these reports, however, relationships between the changes in the respiratory patterns and oxygen intake have not been discussed.

This study was designed to assess changes in the respiratory movement patterns caused by exercise from some viewpoints including energy efficiency. In the present experiment, moderate exercise was adopted, so that the participants could control breathing voluntarily; changes in the respiratory patterns would be induced by exercise, but all the changes would not be physiologically restricted with exercise of this intensity. According to conventional studies on an analysis of the respiratory patterns during exercise, however, respiration rate may be regulated by the rhythm of exercise itself during exercise ${ }^{7)}$. Therefore, in this study, we analyzed the respiratory patterns in the post-exercise recovery phase, in which the participants could control breathing more voluntarily.

\section{Methods}

\section{Participants}

The participants were 15 healthy men (age: 19-33 years; mean age, 22.1 years). The purpose and methods of the present experiment were explained to the participants in advance, and their consent to cooperation as participants of the study was obtained.

\section{Instruments}

A bicycle ergometer (COMBI Co., ERGOMETER 232CXL) was used for exercise.

The respiratory movement patterns were measured by a three-dimensional motion analysis system (OXFORD METRICS INC., VICON370). Respiratory gas and heart rate were measured with a respiratory gas analyzer (Benchmark Exercise Test System, Morgan Inc.).

\section{Procedure}

The participants were forbidden to eat, drink, and smoke for 2 hours before the start of the experiment. Each participant was instructed to sit on a chair, and electrocardiographic (ECG) electrodes, markers for VICON, and a mask for respiratory gas analyzer were applied to each participant. ECG electrodes were applied to each participant according to the thoracic bipolar lead method. The VICON markers were applied to the following four sites: sternoxiphoid process (marker 1); the spine (marker 2) at the same level as that of marker 1; the site right above the umbilicus (marker 3 ); and the spine (marker 4) at the same level as that of marker 3.

After the heart rate was confirmed to have been stable by ECG monitoring, the protocols of exercise and measurement as shown in Table 1 were initiated.

The 7 items measured in each trial were: 1) difference in thoracic expansion between the anterior and posterior diameters at the level of the sternoxiphoid process (calculated from the longitudinal distance between VICON markers 1 and 2 in the expiratory and inspiratory phases), 2) difference in abdominal expansion between the anterior and posterior diameters at the level of the site right above the umbilicus (calculated from the longitudinal distance between VICON markers 3 and 4 in the expiratory and inspiratory phases), 3) tidal volume, 4) respiration rate, 5) minute ventilation, 6) oxygen intake, and 7) heart rate.

All measurements were performed on the participants in a standing position. The fixed point was set at the height of the eyes approximately $5 \mathrm{~m}$ ahead. The participants were instructed to watch closely the fixed point during the measurements. For the purpose of stabilizing the posture, a quadripod cane was placed on the right of participant, and each participant was instructed to put his right hand lightly on the grip during the measurements.

The participants were instructed to keep the standing position even during the intervals between trials after exercise. On this occasion, each participant was instructed to turn his eyes from the fixed point, to let go his hand of the cane, and to stand in a comfortable position.

The above-described items 3) through 7) were measured during exercise as well, i.e., for the last 30 seconds before the end of the exercise.

Table 1 The protocols of exercise and measurement

\begin{tabular}{|c|c|}
\hline Time & Protocol \\
\hline $0^{\prime} 00 " \sim 2^{\prime} 30 "$ & sitting on the chair at rest \\
\hline $2^{\prime} 30 "$ 3'00" & $\begin{array}{l}\text { a postural change to a position for the } \\
\text { measurement }\end{array}$ \\
\hline 3'00" 3'30" & $\downarrow$ measurement before exercise (1st trial) \\
\hline $3^{\prime} 30^{\prime \prime} \sim 4^{\prime} 00^{\prime \prime}$ & riding a bicycle ergometer \\
\hline $4^{\prime} 00^{\prime \prime} \sim 88^{\prime} 50^{\prime \prime}$ & exercise $<100 \mathrm{~W}>$ \\
\hline $8 ' 50 "$ 9'00" & $\begin{array}{l}\text { a postural change to a position for the } \\
\text { measurement }\end{array}$ \\
\hline $9^{\prime} 00^{\prime \prime} \sim 99^{\prime} 30^{\prime \prime}$ & $\downarrow$ measurement after exercise (2nd trial) \\
\hline $9^{\prime} 30^{\prime \prime}$ 10'30" & the interval between the trials \\
\hline $10^{\prime} 30^{\prime \prime}$ 11'00" & $\diamond$ measurement after exercise (3rd trial) \\
\hline $11^{\prime} 00^{\prime \prime} \sim 12^{\prime} 00^{\prime \prime}$ & the interval between the trials \\
\hline $12^{\prime} 00^{\prime \prime}$ 12'30" & $\diamond$ measurement after exercise (4th trial) \\
\hline $12^{\prime} 30^{\prime \prime}$ 13'30" & the interval between trials \\
\hline $133^{\prime} 30^{\prime \prime}$ 14'00" & $\checkmark$ measurement after exercise (5th trial) \\
\hline
\end{tabular}




\section{Data analysis}

The above-described items 1) through 7) were analyzed by using each value measured in the 2 nd to the 5 th trials and during the exercise in each participant, which was calculated in terms of the percentages to the values measured in the 1st trial. Respiratory movement patterns were classified from patterns of differences in thoracic and abdominal expansion between the anterior and posterior diameters at the levels of the sternoxiphoid process (chest expansion) and the site right above the umbilicus (abdominal expansion), respectively, of each participant. Rates of changes in chest expansion and abdominal expansion after exercise were investigated in each participant by the two-way analysis of variance (site $(2) \times$ trial (4)). The participants were classified into groups according to the respiratory movement patterns. Then, respiratory gas parameters and heart rate were compared in each trial during and after exercise among the groups by the two-way analysis of variance.

\section{Results}

\section{Analysis of the respiratory movement patterns}

As a result of ANOVA for rates of changes in chest expansion and abdominal expansion in each participant, the participants were classified into the following 4 groups (Fig. 1).
Abdomen Group: the rate of increase in abdominal expansion was constantly significantly higher than that for chest expansion $(n=7)$

Partly Abdomen Group: the rate of increase in abdominal expansion was partly significantly higher than that for chest expansion $(n=2)$

Abdomen-Chest Group: there was no significant difference in rate of increase between abdominal and chest expansion $(n=2)$

Chest Group: the rate of increase in chest expansion was partly significantly higher than that for abdominal expansion $(n=4)$

The two-way analysis of variance among these 4 groups (group (4) $\times$ trial (4)) revealed that the main effect of factors of participant group on the rate of increase in abdominal expansion was significant $(F(3,44)=3.028$, $\mathrm{p}<.05)$ and the main effect of factors of participant group on the rate of increase in chest expansion was also significant $(\mathrm{F}(3,44)=14.714, \mathrm{p}<.001)$. The post-hoc test (Fisher's PLSD) revealed that the rate of increase in abdominal expansion was significantly higher in Partly Abdomen Group than in Abdomen Group ( $\mathrm{p}<.05)$ and Chest Group $(p<.05)$ and that the rate of increase in chest expansion was significantly lower in Abdomen Group than in three other groups $(\mathrm{p}<.001)$.

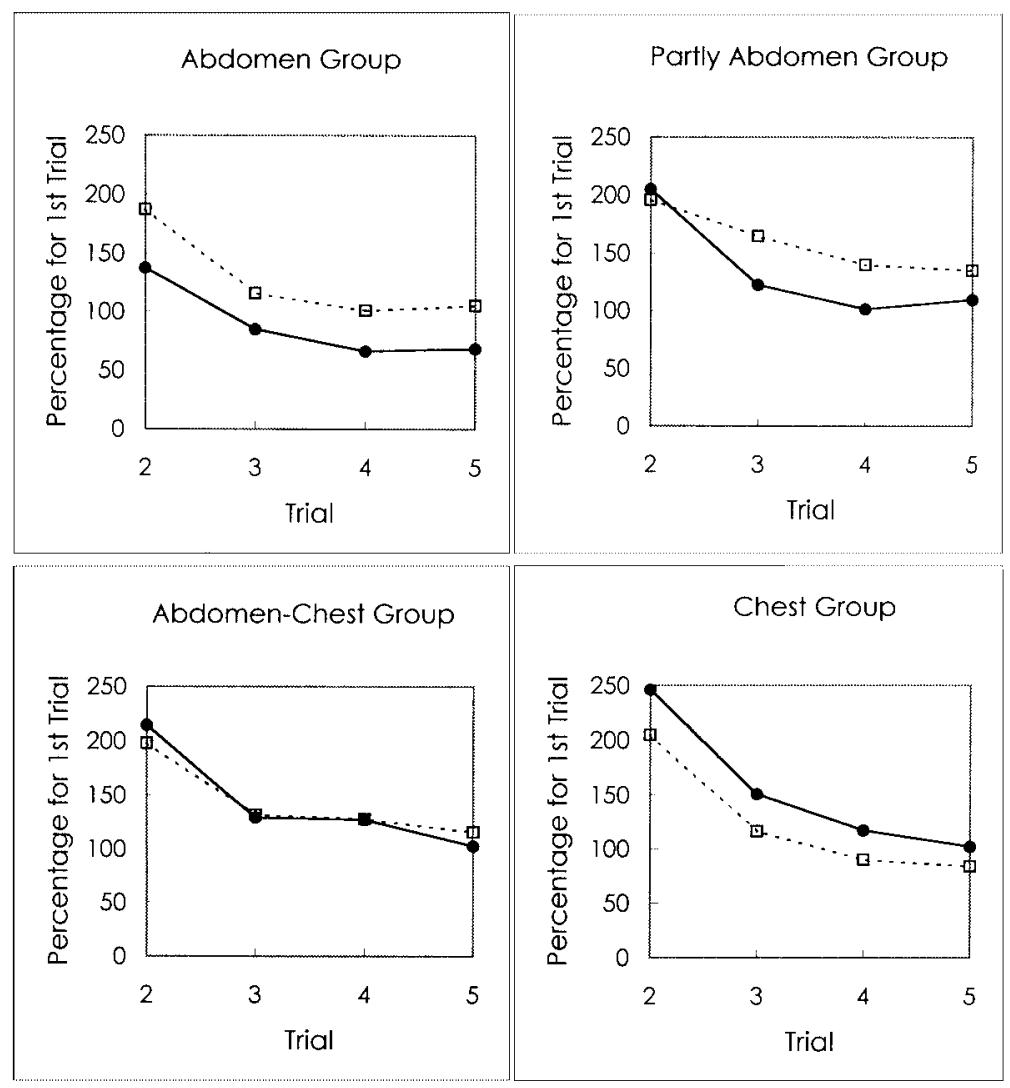

Fig. 1 Pattern of changes in chest expansion and abdominal expansion during post exercise recovery phase. ----: abdominal expansion, Chest expansion. 
Table 2 The mean values of respiratory gas parameters and heart rate in each trial in each group

\begin{tabular}{|c|c|c|c|c|}
\hline & \multicolumn{4}{|c|}{ Percentage for 1st trial (mean \pm SD) } \\
\hline & 2nd trial & 3rd trial & 4th trial & 5th trial \\
\hline \multicolumn{5}{|l|}{ Tidal volume } \\
\hline Abdomen group & $247 \pm 70$ & $126 \pm 32$ & $91 \pm 29$ & $87 \pm 16$ \\
\hline Partly abdomen group & $295 \pm 144$ & $182 \pm 78$ & $135 \pm 29$ & $138 \pm 24$ \\
\hline Abdomen-chest group & $243 \pm 37$ & $128 \pm 39$ & $110 \pm 39$ & $101 \pm 4$ \\
\hline Chest group & $254 \pm 45$ & $127 \pm 26$ & $101 \pm 32$ & $89 \pm 35$ \\
\hline \multicolumn{5}{|l|}{ Respiration rate } \\
\hline Abdomen group & $162 \pm 35$ & $147 \pm 37$ & $137 \pm 30$ & $138 \pm 41$ \\
\hline Partly abdomen group & $112 \pm 17$ & $102 \pm 13$ & $96 \pm 1$ & $107 \pm 29$ \\
\hline Abdomen-chest group & $131 \pm 33$ & $115 \pm 7$ & $122 \pm 19$ & $109 \pm 15$ \\
\hline Chest group & $127 \pm 15$ & $136 \pm 30$ & $153 \pm 59$ & $157 \pm 78$ \\
\hline \multicolumn{5}{|l|}{ Minute ventilation } \\
\hline Abdomen group & $470 \pm 93$ & $195 \pm 37$ & $129 \pm 20$ & $146 \pm 42$ \\
\hline Partly abdomen group & $275 \pm 52$ & $111 \pm 22$ & $71 \pm 12$ & $75 \pm 5$ \\
\hline Abdomen-chest group & $321 \pm 87$ & $153 \pm 24$ & $103 \pm 12$ & $114 \pm 32$ \\
\hline Chest group & $331 \pm 36$ & $162 \pm 55$ & $133 \pm 24$ & $121 \pm 31$ \\
\hline \multicolumn{5}{|l|}{ Oxygen intake } \\
\hline Abdomen group & $314 \pm 61$ & $137 \pm 34$ & $96 \pm 19$ & $108 \pm 23$ \\
\hline Partly abdomen group & $245 \pm 9$ & $108 \pm 8$ & $74 \pm 12$ & $73 \pm 24$ \\
\hline Abdomen-chest group & $244 \pm 4$ & $132 \pm 13$ & $85 \pm 4$ & $108 \pm 44$ \\
\hline Chest group & $263 \pm 38$ & $118 \pm 24$ & $88 \pm 16$ & $76 \pm 23$ \\
\hline \multicolumn{5}{|l|}{ Heart rate } \\
\hline Abdomen group & $150 \pm 15$ & $114 \pm 9$ & $114 \pm 6$ & $117 \pm 8$ \\
\hline Partly abdomen group & $173 \pm 64$ & $152 \pm 35$ & $141 \pm 21$ & $139 \pm 21$ \\
\hline Abdomen-chest group & $135 \pm 22$ & $125 \pm 19$ & $128 \pm 13$ & $117 \pm 4$ \\
\hline Chest group & $158 \pm 23$ & $130 \pm 18$ & $130 \pm 17$ & $128 \pm 15$ \\
\hline
\end{tabular}

Analysis of respiratory gas parameters and heart rate

Oxygen intake and heart rate during exercise were compared among the groups. There were no significant differences among the groups. In all participants, the mean oxygen intake and the mean heart rate were $360.9 \% \pm 62.0$ $\%$ and $171.4 \% \pm 28.4 \%$, respectively, of the levels at rest before exercise. The intensity levels of exercise used in the present experiment were 3 4 METs.

Table 2 shows the mean values of tidal volume, respiration rate, minute ventilation, oxygen intake, and heart rate, respectively, in each trial in each group.

Each parameter was investigated by the two-way analysis of variance (group (4) $\times$ trial (4)), and the obtained results are shown below.

Although there was no significant difference in tidal volume among the groups, tidal volume tended to be higher in Partly Abdomen Group than in Abdomen Group and Chest Group ( $\mathrm{p}<.1)$. There were significant differences in respiration rate $(\mathrm{F}(3,44)=3.131, \mathrm{p}<.05)$, minute ventilation $(\mathrm{F}(3,44)=9.968, \mathrm{p}<.001)$, oxygen intake $(\mathrm{F}(3,44)=4.323$, $\mathrm{p}<.01)$, and heart rate $(\mathrm{F}(3,44)=5.662, \mathrm{p}<.01)$ among the groups. As a result of the post-hoc test (Fisher's PSLD), respiration rate was significantly higher in Abdomen Group and Chest Group than in Partly Abdomen Group ( $\mathrm{p}<.05)$. Minute ventilation was significantly higher in Abdomen Group than in three other groups $(\mathrm{p}<.01)$, and the minute ventilation in Chest Group was higher than that in Partly Abdomen Group ( $\mathrm{p}<.05)$. Oxygen intake was significantly higher in Abdomen Group than in Partly Abdomen Group $(\mathrm{p}<.01)$ and Chest Group $(\mathrm{p}<.05)$. Heart rate was significantly lower in Abdomen Group than in Partly Abdomen Group ( $p<.01)$ and Chest Group ( $<$.05), and the heart rate in Abdomen-Chest Group was significantly lower than that in Partly Abdomen Group ( $\mathrm{p}<.01)$.

\section{Discussion}

In the present experiment, patterns of changes in thoracic and abdominal expansion differences between the anterior and posterior diameters were classified into 4 
groups. The characteristics of each group were markedly observed in the 2 nd trial immediately after exercise. The rate of increase in abdominal expansion was approximately $200 \%$ of that at rest before exercise in each group. On the other hand, the pattern in each group was characterized by the rate of increase in chest expansion. Details of the classification were decided on the basis of differences in the time-course changes.

There were the following relationships between changes in thoracic and abdominal expansion patterns and respiratory gas parameters: tidal volume tended to be higher in Partly Abdomen Group than in Abdomen Group and Chest Group; the rate of increase in abdominal expansion also tended to be higher in Partly Abdomen Group than in Abdomen Group and Chest Group; the rate of increase in chest expansion was almost the same among Partly Abdomen Group, Abdomen-Chest Group, and Chest Group, and the rate was significantly low only in Abdomen Group. These observations suggest that tidal volume is likely to be increased by abdominal expansion rather than thoracic expansion.

Minute ventilation was markedly hihger in Abdomen Group than in three other groups. The tidal volume in Abdomen Group tended to be lower, whereas the respiration rate was high. Accordingly, the increase in minute ventilation observed in the present experiment may have been attributed to the increase in respiration rate, not to the increase in tidal volume. In general, oxygen intake is considered to correlate well with minute ventilation when the intensity of exercise is moderate ${ }^{3)}$. In the present experiment as well, minute ventilation was the highest in Abdomen Group, and oxygen intake was also higher in Abdomen Group than in Partly Abdomen Group and Chest Group. Conversely, heart rate was lower in Abdomen Group than in Partly Abdomen Group and Chest Group.

The above-described results can be summarized as follows. When healthy persons were subjected to exercise at approximately 3 4 METs level, the abdominal expansion was increased to around $200 \%$ of that at rest before exercise in the recovery phase, leading to the increase in ventilation. On this occasion, the degree of abdominal expansion corresponded to $42.3 \%$ of the mean maximum abdominal expansion of all participants. In spite of the presence of room for expansion range, the abdominal expansion was not increased to more than this range. Then, either the strategy for increasing chest expansion (chest breathing strategy) or the strategy for increasing respiration rate (respiration rate strategy) was selected for increasing ventilation. Under the conditions of the present experiment, however, the chest breathing strategy exerted almost no beneficial effect. In Abdomen Group in which the respiration rate strategy was used, minute ventilation was markedly increased, and along with this, oxygen intake was also increased, thereby rapidly restoring heart rate to the level before exercise. In Chest Group, the respiration rate strategy and chest breathing strategy appeared to have been used together, but as shown in Table 2, the combined use may have been a failure in leading to the early recovery, because respiration rate was increased with the decrease in tidal volume in the trials of the latter half of the experiment.

Physical therapists approach to the respiratory function through respiratory movements. Most studies as to respiration, however, have been carried out from the physiological viewpoint. It is important to clarify the relationships among respiratory movement patterns, oxygen intake, and energy efficiency, which relates the physical therapy approach to the improvement in the physiological respiratory function. The results of the present experiment suggest that there are individual differences in selecting strategies under the circumstances, in which the voluntary control can be selected even for the highly automated breathing of healthy person, and that the strategies which will be beneficial to energy efficiency exist. Respiratory movement control and energy efficiency will be increasingly important from the clinical point of view in the future.

\section{References}

1) Holt K, Jeng SF, Ratcliffe R, et al.: Energetic cost and stability during human walking at the preferred stride frequency. J Mot Behav 27: 164-178, 1995.

2) West JB: Respiratory Physiology (5th ed.). Williams \& Wilkins, Baltimore, 1995, pp 51-69.

3) Åstrand PO, Rodahl K: Text Book of Work Physiology. McGraw-Hill, New York, 1986, pp 249-261.

4) Komuro $T$, Mase $K$, Imura $S$, et al.: Changes of breathing pattern (frequency-tidal volume relation) and rib cageabdominal motion during exercise in normal subjects. Rigakuryohogaku 21: 549-552, 1986 (in Japanese).

5) Gallego J, Benammou S, Vardon G, et al.: Influence of thoracoabdominal pattern of breathing on respiratory resistance. Resp Physiol 108: 143-152, 1997.

6) Kitada A, Tamaki A, Matsuo Y, et al:: Analysis of thoracoabdominal movement during incremental work cycle exercise. Proceedings in the 13th International Congress of WCPT, p 315, 1999.

7) Garlando F, Kohl J, Koller EA, et al.: Effect of coupling the breathing and cycling rhythms on oxygen uptake during bicycle ergometry. Eur J Appl Physiol O 54: 497-501, 1985. 